\title{
QUASIEFFICIENT SOLUTIONS OF MULTICRITERIA LOCATION PROBLEMS WITH RECTILINEAR NORM IN $\mathbb{R}^{3}$
}

\author{
Masamichi Kon* \\ Hirosaki University
}

(Received June 14, 2006; Revised February 18, 2007)

\begin{abstract}
A multicriteria location problem with rectilinear norm in $\mathbb{R}^{3}$ and its quasiefficient solutions are considered. First, we give characterizations of its quasiefficient solutions by using optimal solutions of a minisum location problem and by using the concept of the summary diagram. Next, we propose the Frame Generating Algorithm to find all quasiefficient solutions of the multicriteria location problem.
\end{abstract}

Keywords: Facility planning, location problem, multicriteria problem, rectilinear norm, quasiefficiency

\section{Introduction}

Continuous location problems appear very often in economic models of distribution or logistics, in statistics when one tries to find an estimator from a data set or in pure optimization problems where one looks for the optimizer of a certain function. In many situations, one has conflicting objectives. Then the problem is naturally formulated as a multicriteria location problem. As multicriteria location problems, a number of different models have been developed, and they are distinguished according to types of objectives and distances which measure any two points. For a comprehensive overview, see [11] and references in it. In this area, one of main interests is to find efficient or quasiefficient solutions, which are defined later and are very important concepts of solutions for general multicriteria optimization. In terminology for the efficiency, efficient solutions are also called Pareto optimal solutions, and quasiefficient solutions are also called weak Pareto optimal solutions or weak efficient solutions. In this article, we consider quasiefficient solutions. For comparison, efficient solutions are considered only in this section.

On the other hand, in most of papers concerned with algorithms to find all efficient or quasiefficient solutions, multicriteria location problems are considered in the plane $\mathbb{R}^{2}$ (see, for example, $[2,5,9,11,13]$ ). Few papers deal with such algorithms for multicriteria location problems in $\mathbb{R}^{n}(n \geq 3)$. It is due to algorithmic difficulty. Algorithms to find all efficient solutions of multicriteria location problems with rectilinear norm in $\mathbb{R}^{2}, \mathbb{R}^{3}$ and $\mathbb{R}^{n}(n>3)$ were proposed, respectively, in [2], [6] and [8]. And all quasiefficient solutions of multicriteria location problems with block norm in $\mathbb{R}^{2}$ can be determined by using an algorithm proposed in [5]. Block norm is a class of norms containing rectilinear norm as a special case. In [9], an application to the development of new products is considered for an artificial data set by using efficient solutions of multicriteria location problems with block norm in $\mathbb{R}^{2}$. In [7], a public opinion survey on home education is considered for an actual data set, and new learning contents which are near required learning contents as much as possible are determined by using efficient solutions of multicriteria location problems with 
rectilinear norm in $\mathbb{R}^{2}$ and $\mathbb{R}^{3}$. Because learning contents can be considered products, it is a kind of development of new products. Within our knowledge, it is the first application of location problems to the development of new products for an actual data set. The results in [7] suggest that efficient and quasiefficient solutions of multicriteria location problems with rectilinear norm in $\mathbb{R}^{n}(n \geq 3)$ are important and applicable to the development of new products. In that application, each point is considered a product. The rectilinear norm of the difference between two points is the sum of the differences between scores of the two points. Each score represents the value of some character of a product for a point. Thus, using rectilinear norm to measure the difference between two points seems to be suitable for the human senses. In this article, our main interest is to find all quasiefficient solutions of multicriteria location problems with rectilinear norm in $\mathbb{R}^{3}$.

Given demand points in $\mathbb{R}^{3}$, a problem to locate a new facility in $\mathbb{R}^{3}$ is called a single facility location problem. The problem is usually formulated as a minimization problem with an objective function involving distances between the facility and demand points. It is assumed that $m$ demand points $\boldsymbol{d}_{i} \equiv\left(d_{i}^{1}, d_{i}^{2}, d_{i}^{3}\right)^{T} \in \mathbb{R}^{3}, i \in M \equiv\{1,2, \cdots, m\}$ and rectilinear norm $\|\cdot\|_{1}$ defined on $\mathbb{R}^{3}$ are given, where the symbol " $\equiv$ " means that "is defined by". Let $\boldsymbol{x} \equiv\left(x^{1}, x^{2}, x^{3}\right)^{T} \in \mathbb{R}^{3}$ be the location of the facility to be determined. We put $D$ $\equiv\left\{\boldsymbol{d}_{i}: i \in M\right\}$. Without loss of generality, it is assumed that $D \not \subset\left\{\left(x^{1}, x^{2}, x^{3}\right)^{T} \in \mathbb{R}^{3}: x^{j}\right.$ $\left.=x_{0}\right\}$ for each $j \in J \equiv\{1,2,3\}$ and any $x_{0} \in \mathbb{R}$. If its assumption is not satisfied, then our three-dimensional problems reduce to one or two-dimensional problems as in $[2,3,5]$. Our main problem is a multicriteria location problem formulated as follows:

$$
\min _{\boldsymbol{x} \in \mathbb{R}^{3}} \boldsymbol{f}(\boldsymbol{x}) \equiv\left(\left\|\boldsymbol{x}-\boldsymbol{d}_{1}\right\|_{1},\left\|\boldsymbol{x}-\boldsymbol{d}_{2}\right\|_{1}, \cdots,\left\|\boldsymbol{x}-\boldsymbol{d}_{m}\right\|_{1}\right)^{T} .
$$

(P) is a problem to find an efficient or quasiefficient solution. A point $\boldsymbol{x}_{0} \in \mathbb{R}^{3}$ is called an efficient solution of (P) if there is no $\boldsymbol{x} \in \mathbb{R}^{3}$ such that $\boldsymbol{f}(\boldsymbol{x}) \leq \boldsymbol{f}\left(\boldsymbol{x}_{0}\right)$ and $\boldsymbol{f}(\boldsymbol{x}) \neq \boldsymbol{f}\left(\boldsymbol{x}_{0}\right)$, where $\boldsymbol{f}(\boldsymbol{x}) \leq \boldsymbol{f}\left(\boldsymbol{x}_{0}\right)$ means that $\left\|\boldsymbol{x}-\boldsymbol{d}_{i}\right\|_{1} \leq\left\|\boldsymbol{x}_{0}-\boldsymbol{d}_{i}\right\|_{1}$ for all $i \in M$. A point $\boldsymbol{x}_{0} \in \mathbb{R}^{3}$ is called a quasiefficient solution of (P) if there is no $\boldsymbol{x} \in \mathbb{R}^{3}$ such that $\boldsymbol{f}(\boldsymbol{x})<\boldsymbol{f}\left(\boldsymbol{x}_{0}\right)$, where $\boldsymbol{f}(\boldsymbol{x})<\boldsymbol{f}\left(\boldsymbol{x}_{0}\right)$ means that $\left\|\boldsymbol{x}-\boldsymbol{d}_{i}\right\|_{1}<\left\|\boldsymbol{x}_{0}-\boldsymbol{d}_{i}\right\|_{1}$ for all $i \in M$. Let $E(D)$ and $Q E(D)$ be the set of all efficient solutions of $(\mathrm{P})$ and the set of all quasiefficient solutions of $(\mathrm{P})$, respectively. By the above definitions, it can be seen that $D \subset E(D) \subset Q E(D)$. Our main interest is to find $Q E(D)$. In order to characterize quasiefficient solutions of $(\mathrm{P})$, we also consider a minisum location problem formulated as follows:

$$
\min _{\boldsymbol{x} \in \mathbb{R}^{3}} g(\boldsymbol{x}) \equiv \sum_{i=1}^{m} \lambda^{i}\left\|\boldsymbol{x}-\boldsymbol{d}_{i}\right\|_{1}
$$

where each $\lambda^{i}, i \in M$ is a non-negative weight associated with $\boldsymbol{d}_{i}$, and not all $\lambda^{i}$ 's are zero. We put $\boldsymbol{\lambda} \equiv\left(\lambda^{1}, \lambda^{2}, \cdots, \lambda^{m}\right)^{T}$ and denote the set of all optimal solutions of $\left(\mathrm{P}_{\boldsymbol{\lambda}}\right)$ for $\boldsymbol{\lambda}$ as $S^{*}(\boldsymbol{\lambda}) .\left(\mathrm{P}_{\boldsymbol{\lambda}}\right)$ can be solved by using an algorithm proposed in [3].

In section 2, we give some properties of optimal solutions of $\left(\mathrm{P}_{\boldsymbol{\lambda}}\right)$. In section 3 , we give some properties of quasiefficient solutions of $(\mathrm{P})$ by using optimal solutions of $\left(\mathrm{P}_{\boldsymbol{\lambda}}\right)$. In section 4, we propose the Frame Generating Algorithm to find $Q E(D)$, which requires $O\left(m^{4}\right)$ computational time. Finally, we give some conclusions in section 5 .

\section{Optimality of $\left(\mathbf{P}_{\lambda}\right)$}

In this section, we give some properties of optimal solutions of $\left(\mathrm{P}_{\boldsymbol{\lambda}}\right)$.

The following theorem gives the relation between quasiefficient solutions of $(\mathrm{P})$ and optimal solutions of $\left(\mathrm{P}_{\boldsymbol{\lambda}}\right)$. 
Theorem 2.1 ([10]) A point $\boldsymbol{x}_{0} \in \mathbb{R}^{3}$ is quasiefficient in (P) if and only if $\boldsymbol{x}_{0}$ is optimal in $\left(\mathrm{P}_{\boldsymbol{\lambda}}\right)$ for some $\boldsymbol{\lambda} \geq \mathbf{0}$ with $\boldsymbol{\lambda} \neq \mathbf{0}$.

From Theorem 2.1, $Q E(D)$ can be expressed as

$$
Q E(D)=\left\{\boldsymbol{x}^{*} \in \mathbb{R}^{3}: \boldsymbol{x}^{*} \in S^{*}(\boldsymbol{\lambda}) \text { for some } \boldsymbol{\lambda} \geq \mathbf{0} \text { with } \boldsymbol{\lambda} \neq \mathbf{0}\right\} .
$$

Thus, in the following, we investigate properties of optimal solutions of $\left(\mathrm{P}_{\boldsymbol{\lambda}}\right)$.

Since the objective function of $\left(\mathrm{P}_{\boldsymbol{\lambda}}\right), g$, can be rewritten as

$$
g(\boldsymbol{x})=\sum_{i=1}^{m} \lambda^{i}\left\|\boldsymbol{x}-\boldsymbol{d}_{i}\right\|_{1}=\sum_{i=1}^{m} \lambda^{i} \sum_{j=1}^{3}\left|x^{j}-d_{i}^{j}\right|=\sum_{j=1}^{3} \sum_{i=1}^{m} \lambda^{i}\left|x^{j}-d_{i}^{j}\right|,
$$

$\left(\mathrm{P}_{\boldsymbol{\lambda}}\right)$ reduces to three independent one-dimensional problems. Namely, $\boldsymbol{x}^{*} \equiv\left(x^{1 *}, x^{2 *}, x^{3 *}\right)^{T}$ $\in S^{*}(\boldsymbol{\lambda})$ if and only if each $x^{j *}, j \in J$ is an optimal solution of the following one-dimensional problem:

$$
\min _{x \in \mathbb{R}} g_{j}(x) \equiv \sum_{i=1}^{m} \lambda^{i}\left|x-d_{i}^{j}\right|
$$

For each $j \in J$, we denote the set of all optimal solutions of $\left(\mathrm{P}_{j}\right)$ for $\boldsymbol{\lambda}$ as $S_{j}^{*}(\boldsymbol{\lambda})$. In the following, we concentrate on $\left(\mathrm{P}_{1}\right)$. In other $\left(\mathrm{P}_{j}\right), j \in\{2,3\}$, we have the same results as in $\left(\mathrm{P}_{1}\right)$.

Let $f: \mathbb{R} \rightarrow \mathbb{R}$ be a convex function. We denote its left and right derivatives and subdifferential, respectively, as $\frac{d f(x)}{d x^{-}}, \frac{d f(x)}{d x^{+}}$and $\partial f(x)$. Namely,

$$
\frac{d f(x)}{d x^{-}}=\lim _{\alpha \uparrow 0} \frac{f(x+\alpha)-f(x)}{\alpha}, \quad \frac{d f(x)}{d x^{+}}=\lim _{\alpha \downarrow 0} \frac{f(x+\alpha)-f(x)}{\alpha}
$$

and

$$
\partial f(x)=\left[\frac{d f(x)}{d x^{-}}, \frac{d f(x)}{d x^{+}}\right] \equiv\left\{y \in \mathbb{R}: \frac{d f(x)}{d x^{-}} \leq y \leq \frac{d f(x)}{d x^{+}}\right\} .
$$

Note that if $f$ is differentiable at $x_{0}$, then $\partial f\left(x_{0}\right)=\left\{\frac{d f\left(x_{0}\right)}{d x}\right\}$, and that $x_{0}$ minimizes $f$ over $\mathbb{R}$ if and only if $0 \in \partial f\left(x_{0}\right)$ (see, for example, $[4,12]$ ).

For $x \in \mathbb{R}$, we put $L(x) \equiv\left\{i \in M: d_{i}^{1}<x\right\}, R(x) \equiv\left\{i \in M: d_{i}^{1}>x\right\}$ and $I(x) \equiv\{i$ $\left.\in M: d_{i}^{1}=x\right\}$. The objective function of $\left(\mathrm{P}_{1}\right), g_{1}$, is a piecewise linear convex function. It is not differentiable only at each $d_{k}^{1}, k \in M$ with $\lambda^{k}>0$, and we have

$$
\left.\frac{d g_{1}(x)}{d x^{-}}\right|_{x=d_{k}^{1}}=\sum_{i \in L\left(d_{k}^{1}\right)} \lambda^{i}-\sum_{i \in R\left(d_{k}^{1}\right) \cup I\left(d_{k}^{1}\right)} \lambda^{i},\left.\quad \frac{d g_{1}(x)}{d x^{+}}\right|_{x=d_{k}^{1}}=\sum_{i \in L\left(d_{k}^{1}\right) \cup I\left(d_{k}^{1}\right)} \lambda^{i}-\sum_{i \in R\left(d_{k}^{1}\right)} \lambda^{i}
$$

and

$$
\partial g_{1}\left(d_{k}^{1}\right)=\left[\sum_{i \in L\left(d_{k}^{1}\right)} \lambda^{i}-\sum_{i \in R\left(d_{k}^{1}\right) \cup I\left(d_{k}^{1}\right)} \lambda^{i}, \quad \sum_{i \in L\left(d_{k}^{1}\right) \cup I\left(d_{k}^{1}\right)} \lambda^{i}-\sum_{i \in R\left(d_{k}^{1}\right)} \lambda^{i}\right] .
$$

Note that for $k, \ell \in M$ with $d_{k}^{1}<d_{\ell}^{1}, \lambda^{k}>0, \lambda^{\ell}>0$, if there is no $i \in M$ such that $d_{i}^{1} \in\left(d_{k}^{1}, d_{\ell}^{1}\right) \equiv\left\{y \in \mathbb{R}: d_{k}^{1}<y<d_{\ell}^{1}\right\}$ and $\lambda^{i}>0$, then

$$
\left.\frac{d g_{1}(x)}{d x^{+}}\right|_{x=d_{k}^{1}}=\left.\frac{d g_{1}(x)}{d x^{-}}\right|_{x=d_{\ell}^{1}}=\frac{d g_{1}(x)}{d x}, \quad x \in\left(d_{k}^{1}, d_{\ell}^{1}\right) .
$$

We put $d_{\min } \equiv \min \left\{d_{i}^{1}: i \in M\right\}$ and $d_{\max } \equiv \max \left\{d_{i}^{1}: i \in M\right\}$. From $(2), \frac{d g_{1}(x)}{d x}=-\sum_{i=1}^{m} \lambda^{i}$ $<0$ for $x<d_{\min }$ and $\frac{d g_{1}(x)}{d x}=\sum_{i=1}^{m} \lambda^{i}>0$ for $x>d_{\max }$. Thus, we have the following lemma. 
Lemma 2.1 For any fixed $\boldsymbol{\lambda} \geq \mathbf{0}$ with $\boldsymbol{\lambda} \neq \mathbf{0}, S_{1}^{*}(\boldsymbol{\lambda}) \subset\left[d_{\min }, d_{\max }\right]$.

From (2), (3) and Lemma 2.1, (i) $S_{1}^{*}(\boldsymbol{\lambda})=\left\{d_{k}^{1}\right\}$ for some $d_{k}^{1}, k \in M$; or (ii) $S_{1}^{*}(\boldsymbol{\lambda})=\left[d_{k}^{1}, d_{\ell}^{1}\right]$ for some $k, \ell \in M$ such that $d_{k}^{1}<d_{\ell}^{1}, \lambda^{k}>0, \lambda^{\ell}>0$ and that $d_{i}^{1} \notin\left(d_{k}^{1}, d_{\ell}^{1}\right)$ for all $i \in M$ with $\lambda^{i}>0$.

\section{Properties of Quasiefficient Solutions}

In this section, we give some properties of quasiefficient solutions of $(\mathrm{P})$ by using properties of optimal solutions of $\left(\mathrm{P}_{\boldsymbol{\lambda}}\right)$.

For $\boldsymbol{x}_{0} \equiv\left(x_{0}^{1}, x_{0}^{2}, x_{0}^{3}\right)^{T} \in \mathbb{R}^{3}, \boldsymbol{x}_{0}$ is called an intersection point if $x_{0}^{j} \in\left\{d_{i}^{j}: i \in M\right\}, j \in$ $J$. We denote the set of all intersection points as $I$, and put

$$
d_{\min }^{j} \equiv \min \left\{d_{i}^{j}: i \in M\right\}, d_{\max }^{j} \equiv \max \left\{d_{i}^{j}: i \in M\right\}, \quad j \in J .
$$

Then

$$
B_{D} \equiv\left\{\left(x^{1}, x^{2}, x^{3}\right)^{T} \in \mathbb{R}^{3}: d_{\min }^{j} \leq x^{j} \leq d_{\max }^{j}, j \in J\right\}
$$

is called the intersection box (see Figure 1). From Theorem 2.1 and Lemma 2.1, $Q E(D) \subset$ $B_{D}$.

We put $\boldsymbol{e}_{1} \equiv(1,0,0)^{T}, \boldsymbol{e}_{2} \equiv(0,1,0)^{T}, \boldsymbol{e}_{3} \equiv(0,0,1)^{T} \in \mathbb{R}^{3}$ and $\boldsymbol{x}_{k} \equiv\left(x_{k}^{1}, x_{k}^{2}, x_{k}^{3}\right)^{T}$, $\boldsymbol{x}_{h} \equiv\left(x_{h}^{1}, x_{h}^{2}, x_{h}^{3}\right)^{T} \in I$. For each $j \in J$, we call $\boldsymbol{x}_{k}$ an $\boldsymbol{e}_{j}$-oriented intersection point (resp. $a-\boldsymbol{e}_{j}$-oriented intersection point) adjacent to $\boldsymbol{x}_{h}$ if $x_{k}^{j}>x_{h}^{j}\left(\operatorname{resp} . x_{k}^{j}<x_{h}^{j}\right), x_{k}^{j^{\prime}}=x_{h}^{j^{\prime}}, j^{\prime} \neq$ $j$ and there is no $\boldsymbol{x}_{s} \equiv\left(x_{s}^{1}, x_{s}^{2}, x_{s}^{3}\right)^{T} \in I$ such that $x_{h}^{j}<x_{s}^{j}<x_{k}^{j}\left(\right.$ resp. $\left.x_{k}^{j}<x_{s}^{j}<x_{h}^{j}\right)$.

For each $j \in J$, let $d_{[1]}^{j}, d_{[2]}^{j}, \cdots, d_{\left[m_{j}\right]}^{j}$ be all distinct real numbers among $d_{1}^{j}, d_{2}^{j}, \cdots, d_{m}^{j}$ such that $d_{[1]}^{j}<d_{[2]}^{j}<\cdots<d_{\left[m_{j}\right]}^{j}$, and we put

$$
F_{2 k-1}^{j} \equiv\left\{d_{[k]}^{j}\right\}, \quad k=1,2, \cdots, m_{j}
$$

and

$$
F_{2 k}^{j} \equiv\left[d_{[k]}^{j}, d_{[k+1]}^{j}\right], \quad k=1,2, \cdots, m_{j}-1 .
$$

For each $k_{j} \in\left\{1,2, \cdots, 2 m_{j}-1\right\}, j \in J, F_{k_{1}}^{1} \times F_{k_{2}}^{2} \times F_{k_{3}}^{3}$ is called a box. Moreover, if $k$ numbers are even among $k_{1}, k_{2}, k_{3}$, then $F_{k_{1}}^{1} \times F_{k_{2}}^{2} \times F_{k_{3}}^{3}$ is called $k$-dimensional box (see Figure 1).

For any fixed $\boldsymbol{\lambda} \geq \mathbf{0}$ with $\boldsymbol{\lambda} \neq \mathbf{0}, S^{*}(\boldsymbol{\lambda})=\left(F_{k_{1}}^{1} \cup \cdots \cup F_{k_{1}^{\prime}}^{1}\right) \times\left(F_{k_{2}}^{2} \cup \cdots \cup F_{k_{2}^{\prime}}^{2}\right) \times\left(F_{k_{3}}^{3}\right.$ $\left.\cup \cdots \cup F_{k_{3}^{\prime}}^{3}\right)$ for some $k_{j}, k_{j}^{\prime} \in\left\{1,2, \cdots, 2 m_{j}-1\right\}$ with $k_{j} \leq k_{j}^{\prime}, j \in J$. Therefore, $Q E(D)$ is the union of some boxes. The union of all one-dimensional boxes in $Q E(D)$ is called the frame of $Q E(D)$.

Theorem $3.1([15])$ Let $h_{1}(w)$ and $h_{2}(w)$ be convex functions defined on $\mathbb{R}$, where $h_{i}$ is minimized at $w_{i}, i=1,2$ and $w_{1}<w_{2}$. Then, given any $\bar{w} \in\left[w_{1}, w_{2}\right]$, there exists $\theta \in[0$, 1] such that $\bar{w}$ minimizes $\theta h_{2}(w)+(1-\theta) h_{1}(w)$.

In the following corollary, recall that $B_{D}$ is the intersection box defined by (4).

Corollary 3.1 For $\boldsymbol{x}_{1} \equiv\left(x_{1}^{1}, x_{1}^{2}, x_{1}^{3}\right)^{T}, \boldsymbol{x}_{2} \equiv\left(x_{2}^{1}, x_{2}^{2}, x_{2}^{3}\right)^{T} \in B_{D}$, it is assumed that $x_{1}^{j_{0}} \neq$ $x_{2}^{j_{0}}$ for some $j_{0} \in J$ and $x_{1}^{j}=x_{2}^{j}, j \neq j_{0}$. We put $\boldsymbol{x}^{*} \equiv \alpha \boldsymbol{x}_{1}+(1-\alpha) \boldsymbol{x}_{2}, \alpha \in(0,1)$. If $\boldsymbol{x}_{1}$, $\boldsymbol{x}_{2} \in Q E(D)$, then $\boldsymbol{x}^{*} \in Q E(D)$. 


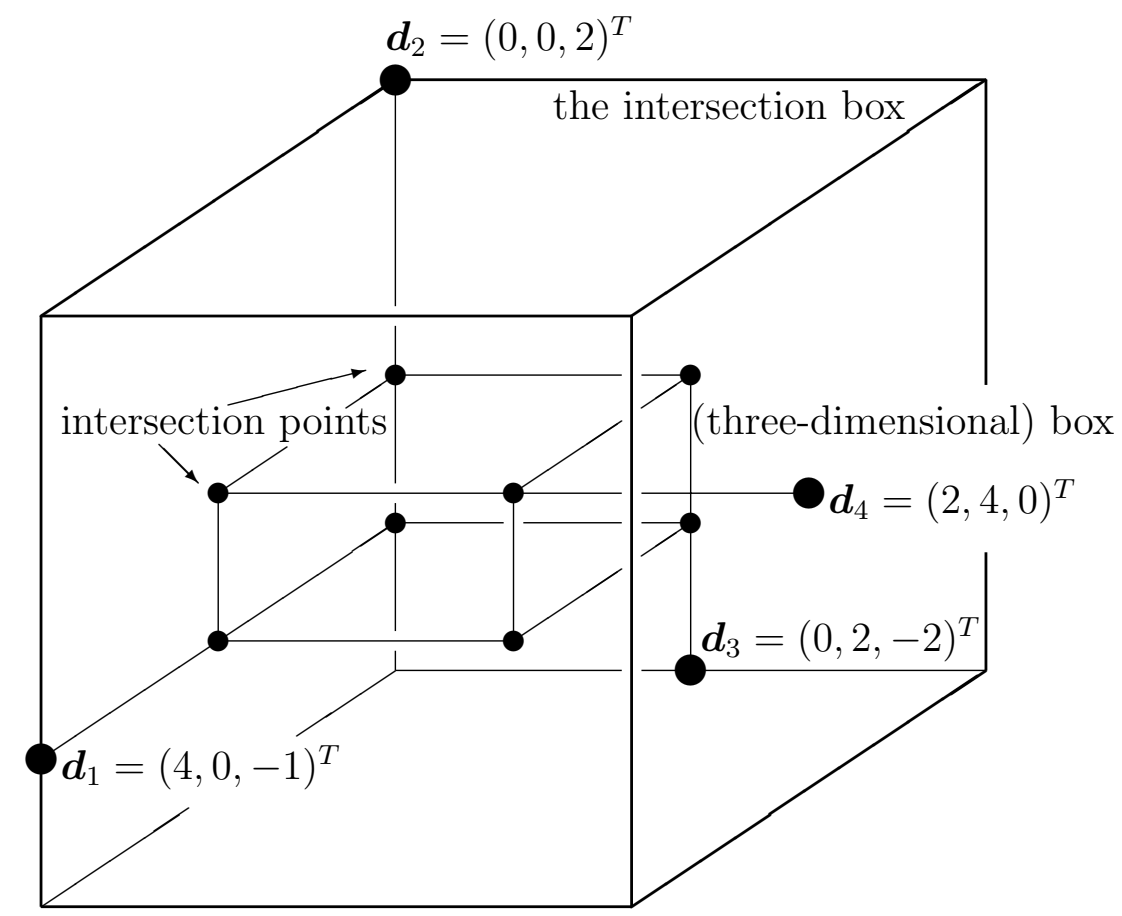

Figure 1: Intersection points, the intersection box, a box

Proof. Without loss of generality, we assume that

$$
x_{1}^{1}<x_{2}^{1}, \quad x_{1}^{j}=x_{2}^{j}, j \in\{2,3\} .
$$

Since $\boldsymbol{x}_{1}, \boldsymbol{x}_{2} \in Q E(D), \boldsymbol{x}_{1} \in S^{*}\left(\boldsymbol{\lambda}_{1}\right)$ and $\boldsymbol{x}_{2} \in S^{*}\left(\boldsymbol{\lambda}_{2}\right)$ for some $\boldsymbol{\lambda}_{1} \geq \mathbf{0}$ and $\boldsymbol{\lambda}_{2} \geq \mathbf{0}$ with $\boldsymbol{\lambda}_{1} \neq \mathbf{0}$ and $\boldsymbol{\lambda}_{2} \neq \mathbf{0}$ by Theorem 2.1. Thus, $x_{1}^{1} \in S_{1}^{*}\left(\boldsymbol{\lambda}_{1}\right), x_{2}^{1} \in S_{1}^{*}\left(\boldsymbol{\lambda}_{2}\right)$ and $x_{1}^{j}, x_{2}^{j} \in S_{j}^{*}\left(\boldsymbol{\lambda}_{1}\right)$ $\bigcap S_{j}^{*}\left(\boldsymbol{\lambda}_{2}\right), j \in\{2,3\}$. For each $j \in\{2,3\}$, if we put $x^{j *} \equiv \alpha x_{1}^{j}+(1-\alpha) x_{2}^{j}$, then $x^{j *} \in$ $S_{j}^{*}\left(\theta \boldsymbol{\lambda}_{1}+(1-\theta) \boldsymbol{\lambda}_{2}\right)$ for any $\theta \in[0,1]$. We put $x^{1 *} \equiv \alpha x_{1}^{1}+(1-\alpha) x_{2}^{1}$. Since $x_{1}^{1}<x^{1 *}<$ $x_{2}^{1}$, there exists $\theta_{1} \in[0,1]$ such that $x^{1 *} \in S_{1}^{*}\left(\theta_{1} \boldsymbol{\lambda}_{1}+\left(1-\theta_{1}\right) \boldsymbol{\lambda}_{2}\right)$ by Theorem 3.1. Since $\boldsymbol{x}^{*}=\left(x^{1 *}, x^{2 *}, x^{3 *}\right)^{T} \in S^{*}\left(\theta_{1} \boldsymbol{\lambda}_{1}+\left(1-\theta_{1}\right) \boldsymbol{\lambda}_{2}\right), \boldsymbol{x}^{*} \in Q E(D)$ by Theorem 2.1.

By Corollary 3.1, if all vertices of a box are quasiefficient in $(\mathrm{P})$, then any point in the box is quasiefficient in $(\mathrm{P})$.

Theorem $3.2([6])$ Let $h_{1}(w)$ and $h_{2}(w)$ be convex functions defined on $\mathbb{R}$, where $h_{i}$ is minimized at $w_{i}, i=1,2$ and $w_{1} \leq w_{2}$. Then, given any $\bar{w} \in\left[w_{1}, w_{2}\right]$,

$$
\left\{\theta \in[0,1]: \bar{w} \text { minimizes } \theta h_{2}(w)+(1-\theta) h_{1}(w)\right\}
$$

is a closed interval.

In the following corollary, recall that $I$ is the set of all intersection points defined before. Corollary 3.2 For $\boldsymbol{x}_{h}=\left(x_{h}^{1}, x_{h}^{2}, x_{h}^{3}\right)^{T}, \boldsymbol{x}_{k}=\left(x_{k}^{1}, x_{k}^{2}, x_{k}^{3}\right)^{T} \in Q E(D) \cap I$, it is assumed that $J_{0} \equiv\left\{j \in J: x_{h}^{j} \neq x_{k}^{j}\right\} \neq \emptyset$. For each $j \in J_{0}$, let $\boldsymbol{y}_{j} \equiv\left(y_{j}^{1}, y_{j}^{2}, y_{j}^{3}\right)^{T}$ be an $\boldsymbol{e}_{j}$-oriented intersection point (resp. a $-\boldsymbol{e}_{j}$-oriented intersection point) adjacent to $\boldsymbol{x}_{k}$ if $x_{h}^{j}>x_{k}^{j}$ (resp. $\left.x_{h}^{j}<x_{k}^{j}\right)$. Then there exists $j_{0} \in J_{0}$ such that $\boldsymbol{y}_{j_{0}} \in Q E(D)$.

Proof. Without loss of generality, we assume that $x_{h}^{j}<x_{k}^{j}, j=1, \cdots, s(\leq 3)$ and $x_{h}^{j}=$ $x_{k}^{j}, j=s+1, \cdots, 3$. We shall show only the case $x_{h}^{1}<x_{k}^{1}, x_{h}^{2}<x_{k}^{2}$ and $x_{h}^{3}=x_{k}^{3}$. In other cases, it can be shown similarly. 
We show that there exists $j_{0} \in J_{0}=\{1,2\}$ such that $\boldsymbol{y}_{j_{0}} \in Q E(D)$, where each $\boldsymbol{y}_{j}=$ $\left(y_{j}^{1}, y_{j}^{2}, y_{j}^{3}\right)^{T}, j \in J_{0}$ is a $-\boldsymbol{e}_{j}$-oriented intersection point adjacent to $\boldsymbol{x}_{k}$. Since $\boldsymbol{x}_{h}, \boldsymbol{x}_{k} \in$ $Q E(D), \boldsymbol{x}_{h} \in S^{*}\left(\boldsymbol{\lambda}_{h}\right)$ and $\boldsymbol{x}_{k} \in S^{*}\left(\boldsymbol{\lambda}_{k}\right)$ for some $\boldsymbol{\lambda}_{h} \equiv\left(\lambda_{h}^{1}, \lambda_{h}^{2}, \cdots, \lambda_{h}^{m}\right)^{T} \geq \mathbf{0}$ and $\boldsymbol{\lambda}_{k} \equiv\left(\lambda_{k}^{1}\right.$, $\left.\lambda_{k}^{2}, \cdots, \lambda_{k}^{m}\right)^{T} \geq \mathbf{0}$ with $\boldsymbol{\lambda}_{h} \neq \mathbf{0}$ and $\boldsymbol{\lambda}_{k} \neq \mathbf{0}$ by Theorem 2.1. Then, for any $j \in J, x_{h}^{j} \in$ $S_{j}^{*}\left(\boldsymbol{\lambda}_{h}\right)$ and $x_{k}^{j} \in S_{j}^{*}\left(\boldsymbol{\lambda}_{k}\right)$. Since $x_{h}^{1} \in S_{1}^{*}\left(\boldsymbol{\lambda}_{h}\right), x_{k}^{1} \in S_{1}^{*}\left(\boldsymbol{\lambda}_{k}\right)$ and $x_{h}^{1} \leq y_{1}^{1} \leq x_{k}^{1},\{\theta \in[0,1]$ : $\left.y_{1}^{1} \in S_{1}^{*}\left(\theta \boldsymbol{\lambda}_{h}+(1-\theta) \boldsymbol{\lambda}_{k}\right)\right\}$ is a nonempty closed interval by Theorem 3.1 and 3.2. Thus, there exists

$$
\theta_{1} \equiv \min \left\{\theta \in[0,1]: y_{1}^{1} \in S_{1}^{*}\left(\theta \boldsymbol{\lambda}_{h}+(1-\theta) \boldsymbol{\lambda}_{k}\right)\right\}
$$

Then we shall show that $x_{k}^{1} \in S_{1}^{*}\left(\delta \boldsymbol{\lambda}_{h}+(1-\delta) \boldsymbol{\lambda}_{k}\right)$ for any $\delta \in\left[0, \theta_{1}\right]$. It is trivial when $\theta_{1}$ $=0$. Thus, we assume that $\theta_{1}>0$. We put

$$
\eta \equiv \frac{\delta}{\theta_{1}} \quad \text { and } \quad \boldsymbol{\lambda}_{1} \equiv \theta_{1} \boldsymbol{\lambda}_{h}+\left(1-\theta_{1}\right) \boldsymbol{\lambda}_{k}
$$

Then

$$
\delta \boldsymbol{\lambda}_{h}+(1-\delta) \boldsymbol{\lambda}_{k}, \quad \delta \in\left[0, \theta_{1}\right]
$$

can be expressed as

$$
\eta \theta_{1} \boldsymbol{\lambda}_{h}+\left(1-\eta \theta_{1}\right) \boldsymbol{\lambda}_{k}=\eta \boldsymbol{\lambda}_{1}+(1-\eta) \boldsymbol{\lambda}_{k}, \quad \eta \in[0,1]
$$

We put

$$
f_{1}(x) \equiv \sum_{i=1}^{m} \lambda_{1}^{i}\left|x-d_{i}^{1}\right| \quad \text { and } \quad f_{k}(x) \equiv \sum_{i=1}^{m} \lambda_{k}^{i}\left|x-d_{i}^{1}\right|
$$

where $\lambda_{1}^{i} \equiv \theta_{1} \lambda_{h}^{i}+\left(1-\theta_{1}\right) \lambda_{k}^{i}, i \in M$. Since $y_{1}^{1} \in S_{1}^{*}\left(\boldsymbol{\lambda}_{1}\right)$ and $x_{k}^{1} \in S_{1}^{*}\left(\boldsymbol{\lambda}_{k}\right), \partial f_{1}\left(y_{1}^{1}\right)=\left[a_{1}\right.$, $\left.a_{2}\right]$ and $\partial f_{k}\left(x_{k}^{1}\right)=\left[b_{1}, b_{2}\right]$ for some $a_{1}, a_{2}, b_{1}, b_{2} \in \mathbb{R}$ such that $a_{1} \leq 0 \leq a_{2}$ and $b_{1} \leq 0 \leq$ $b_{2}$. Since $y_{1}^{1}<x_{k}^{1}, \partial f_{1}\left(x_{k}^{1}\right)=\left[a_{1}^{\prime}, a_{2}^{\prime}\right]$ and $\partial f_{k}\left(y_{1}^{1}\right)=\left[b_{1}^{\prime}, b_{2}^{\prime}\right]$ for some $a_{1}^{\prime}, a_{2}^{\prime}, b_{1}^{\prime}, b_{2}^{\prime} \in \mathbb{R}$ such that $a_{2} \leq a_{1}^{\prime} \leq a_{2}^{\prime}$ and $b_{1}^{\prime} \leq b_{2}^{\prime} \leq b_{1}$. For $\eta \in[0,1]$, we put

$$
G(\eta) \equiv \eta \partial f_{1}\left(x_{k}^{1}\right)+(1-\eta) \partial f_{k}\left(x_{k}^{1}\right)=\left[b_{1}+\eta\left(a_{1}^{\prime}-b_{1}\right), b_{2}+\eta\left(a_{2}^{\prime}-b_{2}\right)\right]
$$

and

$$
H(\eta) \equiv \eta \partial f_{1}\left(y_{1}^{1}\right)+(1-\eta) \partial f_{k}\left(y_{1}^{1}\right)=\left[b_{1}^{\prime}+\eta\left(a_{1}-b_{1}^{\prime}\right), b_{2}^{\prime}+\eta\left(a_{2}-b_{2}^{\prime}\right)\right] .
$$

By definitions of $\theta_{1}$ and $\eta$ (see (5) and (6)),

$$
0 \in H(1), \quad 0 \notin H(\eta), \eta \in[0,1] \backslash\{1\} .
$$

Thus, it needs that $a_{2}=0$ and $b_{2}^{\prime}<0$. By Theorem 3.1, for $x_{0} \in\left(y_{1}^{1}, x_{k}^{1}\right)$, there exists $\eta_{0} \in$ $[0,1]$ such that $x_{0} \in S_{1}^{*}\left(\eta_{0} \boldsymbol{\lambda}_{1}+\left(1-\eta_{0}\right) \boldsymbol{\lambda}_{k}\right)$. In this case, $\left[y_{1}^{1}, x_{k}^{1}\right] \subset S_{1}^{*}\left(\eta_{0} \boldsymbol{\lambda}_{1}+\left(1-\eta_{0}\right) \boldsymbol{\lambda}_{k}\right)$. Thus, we have

$$
0 \in G\left(\eta_{0}\right) \bigcap H\left(\eta_{0}\right)
$$

From (8), it needs that $\eta_{0}=1$. Since it needs that $a_{1}^{\prime}=0,0 \in G(\eta)$ for any $\eta \in[0,1]$. By the definition of $G(\eta)$ (see (7)), $x_{k}^{1} \in S_{1}^{*}\left(\eta \boldsymbol{\lambda}_{1}+(1-\eta) \boldsymbol{\lambda}_{k}\right)$ for any $\eta \in[0,1]$. Namely, by definitions of $\eta$ and $\boldsymbol{\lambda}_{1}$ (see (6)), $x_{k}^{1} \in S_{1}^{*}\left(\delta \boldsymbol{\lambda}_{h}+(1-\delta) \boldsymbol{\lambda}_{k}\right)$ for any $\delta \in\left[0, \theta_{1}\right]$. Since $x_{h}^{2}$ $\in S_{2}^{*}\left(\boldsymbol{\lambda}_{h}\right), x_{k}^{2} \in S_{2}^{*}\left(\boldsymbol{\lambda}_{k}\right)$ and $x_{h}^{2} \leq y_{2}^{2} \leq x_{k}^{2},\left\{\theta \in[0,1]: y_{2}^{2} \in S_{2}^{*}\left(\theta \boldsymbol{\lambda}_{h}+(1-\theta) \boldsymbol{\lambda}_{k}\right)\right\}$ is a nonempty closed interval by Theorem 3.1 and 3.2. Thus, there exists

$$
\theta_{2} \equiv \min \left\{\theta \in[0,1]: y_{2}^{2} \in S_{2}^{*}\left(\theta \boldsymbol{\lambda}_{h}+(1-\theta) \boldsymbol{\lambda}_{k}\right)\right\}
$$


Repeating the above discussion, it can be shown that $x_{k}^{2} \in S_{2}^{*}\left(\delta \boldsymbol{\lambda}_{h}+(1-\delta) \boldsymbol{\lambda}_{k}\right)$ for any $\delta$ $\in\left[0, \theta_{2}\right]$.

On the other hand, since $x_{k}^{3} \in S_{3}^{*}\left(\boldsymbol{\lambda}_{h}\right) \bigcap S_{3}^{*}\left(\boldsymbol{\lambda}_{k}\right), x_{k}^{3} \in S_{3}^{*}\left(\theta \boldsymbol{\lambda}_{h}+(1-\theta) \boldsymbol{\lambda}_{k}\right)$ for any $\theta \in$ $[0,1]$. We put

$$
j_{0} \equiv\left\{\begin{array}{ll}
1 & \text { if } \theta_{1} \leq \theta_{2}, \\
2 & \text { if } \theta_{1}>\theta_{2}
\end{array} \quad \text { and } \quad \boldsymbol{\lambda}_{j_{0}} \equiv \theta_{j_{0}} \boldsymbol{\lambda}_{h}+\left(1-\theta_{j_{0}}\right) \boldsymbol{\lambda}_{k}\right.
$$

Then $y_{j_{0}}^{j} \in S_{j}^{*}\left(\boldsymbol{\lambda}_{j_{0}}\right), j \neq j_{0}$ and $y_{j_{0}}^{j_{0}} \in S_{j_{0}}^{*}\left(\boldsymbol{\lambda}_{j_{0}}\right)$ by the definition of $j_{0}$ (see (5), (9) and (10)). Since $\boldsymbol{y}_{j_{0}} \in S^{*}\left(\boldsymbol{\lambda}_{j_{0}}\right), \boldsymbol{y}_{j_{0}} \in Q E(D)$ by Theorem 2.1.

From Corollary 3.1 and 3.2, there exists "zig-zag path" between any two quasiefficient solutions of $(\mathrm{P})$. The zig-zag path consists of line segments in $\mathbb{R}^{3}$, each of which is contained in $Q E(D)$ and parallel to some line $\left\{\alpha \boldsymbol{e}_{j}: \alpha \in \mathbb{R}\right\}, j \in J$. If the two quasiefficient solutions of $(\mathrm{P})$ are intersection points, there exists the zig-zag path which consists of one-dimensional boxes in $Q E(D)$. Moreover, the frame of $Q E(D)$, which is the union of all one-dimensional boxes in $Q E(D)$ defined before, is connected. If the frame of $Q E(D)$ is determined, then $Q E(D)$ can be constructed. Thus, we give an algorithm to find the frame of $Q E(D)$ in the next section.

\section{Algorithm to Find All Quasiefficient Solutions}

In this section, we propose the Frame Generating Algorithm to find the frame of $Q E(D)$, which requires $O\left(m^{4}\right)$ computational time.

In the Frame Generating Algorithm, checking that an intersection point is quasiefficient in $(\mathrm{P})$ or not is needed. Thus, in the following, we state how to check it.

For $\boldsymbol{x} \in \mathbb{R}^{3}$, we put

$$
B_{\boldsymbol{d}_{i}}(\boldsymbol{x}) \equiv\left\{\boldsymbol{y} \in \mathbb{R}^{3}:\left\|\boldsymbol{y}-\boldsymbol{d}_{i}\right\|_{1}<\left\|\boldsymbol{x}-\boldsymbol{d}_{i}\right\|_{1}\right\}, i \in M \quad \text { and } \quad B(\boldsymbol{x}) \equiv \bigcap_{i=1}^{m} B_{\boldsymbol{d}_{i}}(\boldsymbol{x}) .
$$

By the definition of the quasiefficiency, $\boldsymbol{x}_{0} \in Q E(D)$ if and only if $B\left(\boldsymbol{x}_{0}\right)=\emptyset$. For $\varepsilon>0$ and $\boldsymbol{x} \in \mathbb{R}^{3}$, we put

$$
D_{\varepsilon}(\boldsymbol{x}) \equiv N_{\varepsilon}(\boldsymbol{x}) \bigcap B(\boldsymbol{x})
$$

where $N_{\varepsilon}(\boldsymbol{x})$ is an $\varepsilon$-neighbourhood of $\boldsymbol{x}$. Then we have the following lemma.

Lemma 4.1 A point $\boldsymbol{x}_{0} \in \mathbb{R}^{3}$ is quasiefficient in $(\mathrm{P})$ if and only if $D_{\varepsilon}\left(\boldsymbol{x}_{0}\right)=\emptyset$ for some $\varepsilon>0$.

Proof. If $\boldsymbol{x}_{0} \in Q E(D)$, then $D_{\varepsilon}\left(\boldsymbol{x}_{0}\right) \subset B\left(\boldsymbol{x}_{0}\right)=\emptyset$ for any $\varepsilon>0$ by the definition of the quasiefficiency.

Assume that $\boldsymbol{x}_{0} \notin Q E(D)$. Then there exists $\boldsymbol{y} \in \mathbb{R}^{3}$ such that $\left\|\boldsymbol{y}-\boldsymbol{d}_{i}\right\|_{1}<\left\|\boldsymbol{x}_{0}-\boldsymbol{d}_{i}\right\|_{1}$, $i \in M$. For $\alpha \in(0,1),\left\|(1-\alpha) \boldsymbol{x}_{0}+\alpha \boldsymbol{y}-\boldsymbol{d}_{i}\right\|_{1}<\left\|\boldsymbol{x}_{0}-\boldsymbol{d}_{i}\right\|_{1}, i \in M$ by the convexity of $\|\cdot\|_{1}$. For any $\varepsilon>0$, if $\alpha$ is sufficiently small, then $(1-\alpha) \boldsymbol{x}_{0}+\alpha \boldsymbol{y} \in D_{\varepsilon}\left(\boldsymbol{x}_{0}\right)$.

Following [6], we introduce the concept of the summary diagram in order to check that an intersection point is quasiefficient in $(\mathrm{P})$ or not by applying Lemma 4.1. In [14], the summary diagram is introduced for multicriteria location problems with one-infinity norm in $\mathbb{R}^{2}$. Then, in [6], it is extended for multicriteria location problems with rectilinear norm 
in $\mathbb{R}^{3}$. We put

$$
\begin{aligned}
& O_{1} \equiv\left\{\left(x^{1}, x^{2}, x^{3}\right)^{T} \in \mathbb{R}^{3}: x^{1} \geq 0, x^{2} \geq 0, x^{3} \geq 0\right\} \\
& O_{2} \equiv\left\{\left(x^{1}, x^{2}, x^{3}\right)^{T} \in \mathbb{R}^{3}: x^{1} \leq 0, x^{2} \geq 0, x^{3} \geq 0\right\} \\
& O_{3} \equiv\left\{\left(x^{1}, x^{2}, x^{3}\right)^{T} \in \mathbb{R}^{3}: x^{1} \leq 0, x^{2} \leq 0, x^{3} \geq 0\right\} \\
& O_{4} \equiv\left\{\left(x^{1}, x^{2}, x^{3}\right)^{T} \in \mathbb{R}^{3}: x^{1} \geq 0, x^{2} \leq 0, x^{3} \geq 0\right\}
\end{aligned}
$$

and

$$
O_{-\eta} \equiv\left\{-\boldsymbol{x}: \boldsymbol{x} \in O_{\eta}\right\}, \quad \eta=1,2,3,4 .
$$

For $\boldsymbol{x} \in \mathbb{R}^{3}$, the summary diagram of $\boldsymbol{x}, S D(\boldsymbol{x})$, is defined as follows:

$$
S D(\boldsymbol{x}) \equiv\left\{\eta \in\{ \pm 1, \pm 2, \pm 3, \pm 4\}: \boldsymbol{d}_{i} \in O_{\eta}(\boldsymbol{x}) \text { for some } i \in M\right\}
$$

where $O_{\eta}(\boldsymbol{x}) \equiv\left\{\boldsymbol{x}+\boldsymbol{y}: \boldsymbol{y} \in O_{\eta}\right\}$ for $\eta \in\{ \pm 1, \pm 2, \pm 3, \pm 4\}$. Conveniently, $S D(\boldsymbol{x})$ is represented in diagram form as follows: First, we draw the cube with vertices $\boldsymbol{v}_{1} \equiv(1,1$, $1)^{T}, \boldsymbol{v}_{2} \equiv(-1,1,1)^{T}, \boldsymbol{v}_{3} \equiv(-1,-1,1)^{T}, \boldsymbol{v}_{4} \equiv(1,-1,1)^{T}$ and $\boldsymbol{v}_{-\eta} \equiv-\boldsymbol{v}_{\eta}, \eta=1,2,3,4$; Next, for each $\eta \in\{ \pm 1, \pm 2, \pm 3, \pm 4\}$, dot $\boldsymbol{v}_{\eta}$ if $\eta \in S D(\boldsymbol{x})$. For example, $S D(\boldsymbol{x})=\{2,3$, $4,-2,-3\}$ for $\boldsymbol{d}_{1}=(3,0,4)^{T}, \boldsymbol{d}_{2}=(4,2,0)^{T}, \boldsymbol{d}_{3}=(2,1,3)^{T}, \boldsymbol{d}_{4}=(0,4,5)^{T}, \boldsymbol{d}_{5}=(1,5,2)^{T}$ and $\boldsymbol{x}=(3,2,1)^{T}$. Figure 2 shows its summary diagram in diagram form.

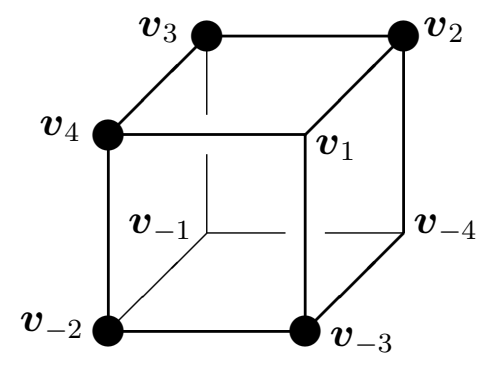

Figure 2: $S D(\boldsymbol{x})=\{2,3,4,-2,-3\}$

In the following, recall again that $I$ is the set of all intersection points defined before. In [6], it is shown that $S D\left(\boldsymbol{x}_{0}\right)$ for $\boldsymbol{x}_{0} \in I$ coincides with one of patterns illustrated in Figure 3 , where we identify summary diagrams in diagram form if they are the same pattern by rotation. Fix any $\boldsymbol{x}_{0} \in I$. Suppose, for example, that $S D\left(\boldsymbol{x}_{0}\right)=\{1,2,4,-2\}$. Then the pattern of $S D\left(\boldsymbol{x}_{0}\right)$ is (xi) in Figure 3. By the definition of the summary diagram (see (12)), $O_{\eta}\left(\boldsymbol{x}_{0}\right) \cap D \neq \emptyset$ for $\eta \in S D\left(\boldsymbol{x}_{0}\right)$. By the definition of $D_{\varepsilon}\left(\boldsymbol{x}_{0}\right)$ (see (11)), we have $D_{\varepsilon}\left(\boldsymbol{x}_{0}\right)$ $\subset\left\{\boldsymbol{x}_{0}+\boldsymbol{x}: \boldsymbol{x} \in \mathbb{R}^{3},\left\langle\boldsymbol{x}, \boldsymbol{v}_{\eta}\right\rangle>0, \eta \in S D\left(\boldsymbol{x}_{0}\right)\right\} \cap N_{\varepsilon}\left(\boldsymbol{x}_{0}\right)=\emptyset$ for $\varepsilon>0$, where $\langle\boldsymbol{x}, \boldsymbol{y}\rangle \equiv$ $x^{1} y^{1}+x^{2} y^{2}+x^{3} y^{3}$ for $\boldsymbol{x}=\left(x^{1}, x^{2}, x^{3}\right)^{T}, \boldsymbol{y}=\left(y^{1}, y^{2}, y^{3}\right)^{T} \in \mathbb{R}^{3}$. Thus, $\boldsymbol{x}_{0} \in Q E(D)$ by Lemma 4.1. Similarly, we have the following results by simple calculations. If the pattern of $S D\left(\boldsymbol{x}_{0}\right)$ is one of (i)-(xi) in Figure 3, then the $D_{\varepsilon}\left(\boldsymbol{x}_{0}\right)=\emptyset$ for $\varepsilon>0$. In this case, $\boldsymbol{x}_{0} \in$ $Q E(D)$ by Lemma 4.1. If the pattern of $S D\left(\boldsymbol{x}_{0}\right)$ is (xii) in Figure 3, then $D_{\varepsilon}\left(\boldsymbol{x}_{0}\right) \neq \emptyset$ for $\varepsilon>0$. In this case, $\boldsymbol{x}_{0} \notin Q E(D)$ by Lemma 4.1. Thus, we have the following theorem and corollary.

Theorem 4.1 For an intersection point $\boldsymbol{x}_{0} \in I, \boldsymbol{x}_{0} \notin Q E(D)$ if and only if the pattern of the summary diagram of $\boldsymbol{x}_{0}$ coincides with the pattern (xii) in Figure 3.

Corollary 4.1 For an intersection point $\boldsymbol{x}_{0} \in I, \boldsymbol{x}_{0} \in Q E(D)$ if and only if $\{ \pm \eta\} \subset S D\left(\boldsymbol{x}_{0}\right)$ for some $\eta \in\{1,2,3,4\}$.

Given $\boldsymbol{x}_{0} \in I$, the pattern of $S D\left(\boldsymbol{x}_{0}\right)$ can be determined in $O(m)$ computational time by comparing components of $\boldsymbol{x}_{0}$ and each $\boldsymbol{d}_{i}, i \in M$. Namely, checking that $\boldsymbol{x}_{0} \in Q E(D)$ or not requires $O(m)$ computational time. 
(i)

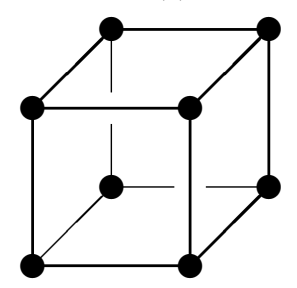

(v)

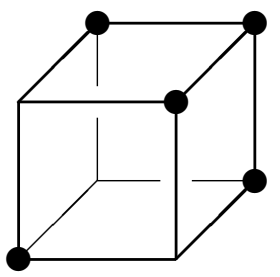

(ix)

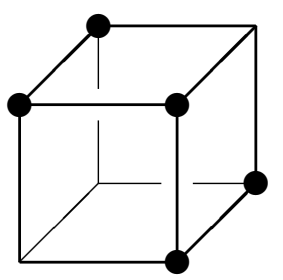

(ii)

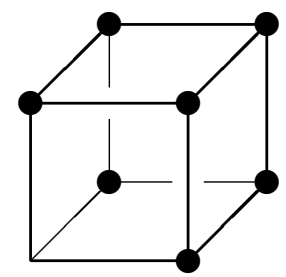

(vi)

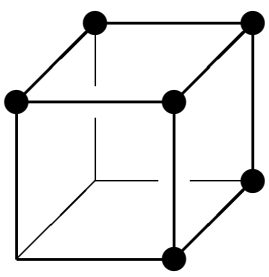

$(\mathrm{x})$

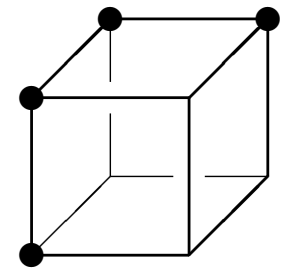

(iii)

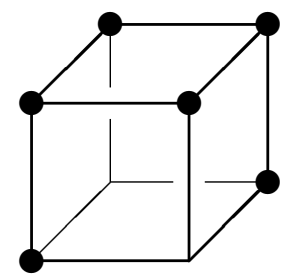

(vii)

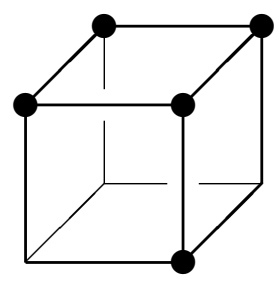

(xi)

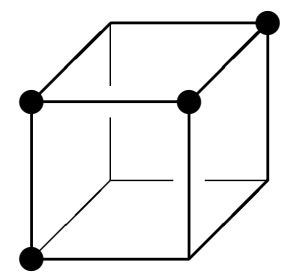

(iv)

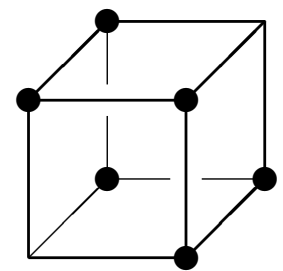

(viii)

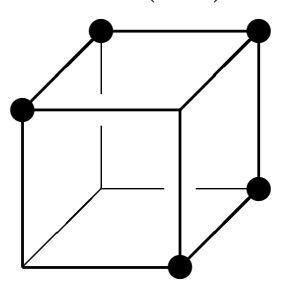

(xii)

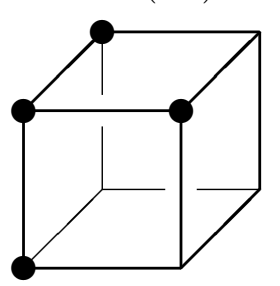

Figure 3: Patterns of summary diagrams of an intersection point

Remark 4.1 In view of the fact that the frame of $Q E(D)$ is the union of all one-dimensional boxes in $Q E(D)$, which is connected, we can construct a connected graph $(V, E)$, where $V$ $=I \bigcap Q E(D)$ and $E$ is the set of all arcs in the graph. Given $\boldsymbol{x}_{1}, \boldsymbol{x}_{2} \in I \cap Q E(D)$, the arc $a\left(\boldsymbol{x}_{1}, \boldsymbol{x}_{2}\right)$ which connects $\boldsymbol{x}_{1}$ and $\boldsymbol{x}_{2}$ is in $E$ if and only if $\boldsymbol{x}_{1}$ and $\boldsymbol{x}_{2}$ are adjacent and quasiefficient in $(\mathrm{P})$. This concept will be the guide for describing an algorithm to locate the frame of $Q E(D)$.

In the Frame Generating Algorithm, we put

$$
V_{i} \equiv\left\{\left(d_{[k]}^{1}, d_{[\ell]}^{2}, d_{[i]}^{3}\right)^{T} \in \mathbb{R}^{3}: k \in\left\{1,2, \cdots, m_{1}\right\}, \ell \in\left\{1,2, \cdots, m_{2}\right\}\right\}, \quad i=1,2, \cdots, m_{3} .
$$

We use $r$ as a counter which represents the number of iterations. For each $r \in\{1,2, \cdots$, $\left.m_{3}\right\}$, the Frame Generating Algorithm finds one-dimensional boxes in the frame of $Q E(D)$, which are connected with any initial point $\boldsymbol{d}_{k_{r}} \in V_{r}$ through only intersection points in $V_{r} \cap Q E(D) . L_{r} \subset V_{r}$ is the set of checked intersection points which are connected with the initial point through only intersection points in $V_{r} \bigcap Q E(D) . S_{r} \subset L_{r}$ is the set of intersection points which have been checked that one-dimensional boxes connected with them are contained $Q E(D)$ or not. $D_{r}, G_{r} \subset V_{r}$ are the sets of checked intersection points which are quasiefficient and not quasiefficient in $(\mathrm{P})$, respectively. We use $T$ as the union of one-dimensional boxes in $Q E(D)$ which have been checked before. Moreover, for $\boldsymbol{x}, \boldsymbol{y} \in \mathbb{R}^{3}$, we put $[\boldsymbol{x}, \boldsymbol{y}] \equiv\{(1-\lambda) \boldsymbol{x}+\lambda \boldsymbol{y}: \lambda \in[0,1]\}$. 


\section{The Frame Generating Algorithm}

Step 0. Set $S_{i}=\emptyset, G_{i}=\emptyset, D_{i}=D \bigcap V_{i}, i=1,2, \cdots, m_{3}$. For each $i \in\left\{1,2, \cdots, m_{3}\right\}$, choose any $\boldsymbol{d}_{k_{i}} \in D_{i}$ and set $L_{i}=\left\{\boldsymbol{d}_{k_{i}}\right\}$. Set $T=\emptyset$ and $r=1$.

Step 1. If $L_{r}=S_{r}$, then set $r=r+1$. If $r>m_{3}$, then stop. ( $T$ is the frame of $Q E(D)$.)

Step 2. Choose any $\boldsymbol{x}_{0}=\left(d_{[k]}^{1}, d_{[\ell]}^{2}, d_{[r]}^{3}\right)^{T} \in L_{r} \backslash S_{r}$ and set $S_{r}=S_{r} \bigcup\left\{\boldsymbol{x}_{0}\right\}$.

Step 3. Set $W=\emptyset$.

(a) If $k>1$, then set $\boldsymbol{x}_{-1}=\left(d_{[k-1]}^{1}, d_{[\ell]}^{2}, d_{[r]}^{3}\right)^{T}$ and $W=W \bigcup\left\{\boldsymbol{x}_{-1}\right\}$.

(b) If $k<m_{1}$, then set $\boldsymbol{x}_{1}=\left(d_{[k+1]}^{1}, d_{[\ell]}^{2}, d_{[r]}^{3}\right)^{T}$ and $W=W \bigcup\left\{\boldsymbol{x}_{1}\right\}$.

(c) If $\ell>1$, then set $\boldsymbol{x}_{-2}=\left(d_{[k]}^{1}, d_{[\ell-1]}^{2}, d_{[r]}^{3}\right)^{T}$ and $W=W \bigcup\left\{\boldsymbol{x}_{-2}\right\}$.

(d) If $\ell<m_{2}$, then set $\boldsymbol{x}_{2}=\left(d_{[k]}^{1}, d_{[\ell+1]}^{2}, d_{[r]}^{3}\right)^{T}$ and $W=W \bigcup\left\{\boldsymbol{x}_{2}\right\}$.

Step 4. If $W=\emptyset$, then go to Step 6 , otherwise choose any $\boldsymbol{x}_{\eta} \in W$ and set $W=W \backslash$ $\left\{\boldsymbol{x}_{\eta}\right\}$.

Step 5. If $\left[\boldsymbol{x}_{0}, \boldsymbol{x}_{\eta}\right] \subset T$, then go to Step 4 .

(a) If $\boldsymbol{x}_{\eta} \in D_{r}$, then set $T=T \bigcup\left[\boldsymbol{x}_{0}, \boldsymbol{x}_{\eta}\right]$, and if $\boldsymbol{x}_{\eta} \notin L_{r}$ then $L_{r}=L_{r} \bigcup\left\{\boldsymbol{x}_{\eta}\right\}$, and go to Step 4.

(b) If $\boldsymbol{x}_{\eta} \notin G_{r}$, then check that $\boldsymbol{x}_{\eta} \in Q E(D)$ or not by using its summary diagram. If $\boldsymbol{x}_{\eta} \in Q E(D)$, then set $T=T \bigcup\left[\boldsymbol{x}_{0}, \boldsymbol{x}_{\eta}\right], D_{r}=D_{r} \bigcup\left\{\boldsymbol{x}_{\eta}\right\}$ and $L_{r}=L_{r} \bigcup\left\{\boldsymbol{x}_{\eta}\right\}$, otherwise set

$$
G_{r}= \begin{cases}G_{r} \bigcup\left\{\left(d_{[p]}^{1}, d_{[\ell]}^{2}, d_{[r]}^{3}\right)^{T}: p=1,2, \cdots, k-1\right\} & \text { if } \eta=-1 \\ G_{r} \bigcup\left\{\left(d_{[p]}^{1}, d_{[\ell]}^{2}, d_{[r]}^{3}\right)^{T}: p=k+1, \cdots, m_{1}\right\} & \text { if } \eta=1, \\ G_{r} \bigcup\left\{\left(d_{[k]}^{1}, d_{[p]}^{2}, d_{[r]}^{3}\right)^{T}: p=1,2, \cdots, \ell-1\right\} & \text { if } \eta=-2 \\ G_{r} \bigcup\left\{\left(d_{[k]}^{1}, d_{[p]}^{2}, d_{[r]}^{3}\right)^{T}: p=\ell+1, \cdots, m_{2}\right\} & \text { if } \eta=2\end{cases}
$$

Go to Step 4.

Step 6. If $r<m_{3}$, then set $\boldsymbol{x}_{3}=\left(d_{[k]}^{1}, d_{[\ell]}^{2}, d_{[r+1]}^{3}\right)^{T}$, otherwise go to Step 1 .

(a) If $\boldsymbol{x}_{3} \in D_{r+1}$, then set $T=T \bigcup\left[\boldsymbol{x}_{0}, \boldsymbol{x}_{3}\right]$ and go to Step 1.

(b) Check that $\boldsymbol{x}_{3} \in Q E(D)$ or not by using its summary diagram. If $\boldsymbol{x}_{3} \in Q E(D)$, then set $T=T \bigcup\left[\boldsymbol{x}_{0}, \boldsymbol{x}_{3}\right]$ and $D_{r+1}=D_{r+1} \bigcup\left\{\boldsymbol{x}_{3}\right\}$, otherwise set $G_{p}=G_{p} \bigcup\left\{\left(d_{[k]}^{1}, d_{[\ell]}^{2}\right.\right.$, $\left.\left.d_{[p]}^{3}\right)^{T}\right\}, p=r+1, \cdots, m_{3}$. Go to Step 1 .

In Step 0, for each $j \in J$, we can obtain $d_{[1]}^{j}, d_{[2]}^{j}, \cdots, d_{\left[m_{j}\right]}^{j}$ by sorting $m$ real numbers $d_{1}^{j}$, $d_{2}^{j}, \cdots, d_{m}^{j}$ which requires $O(m \log m)$ computational time [1]. Then $V_{i}, D_{i}, i=1,2, \cdots, m_{3}$ are determined. Thus, $D_{i}, i=1,2, \cdots, m_{3}$ can be determined in $O(m \log m)$ computational time. In $r$ th iteration, the Frame Generating Algorithm checks that intersection points adjacent to each intersection points in $L_{r} \subset V_{r}$ are quasiefficient in (P) or not by using their summary diagrams. The number of iterations is $O(m)$. The number of intersection points in $L_{r}$ is $O\left(\mathrm{~m}^{2}\right)$ and the number of intersection points adjacent to an intersection point, which should be checked, is at most five. Checking that an intersection point is quasiefficient in $(\mathrm{P})$ or not by using its summary diagram requires $O(m)$ computational time. Therefore, the Frame Generating Algorithm requires $O\left(m^{4}\right)$ computational time.

Finally, we consider an example problem for $\boldsymbol{d}_{1}=(3,0,4)^{T}, \boldsymbol{d}_{2}=(4,2,0)^{T}, \boldsymbol{d}_{3}=(2$, $1,3)^{T}$ and $\boldsymbol{d}_{4}=(0,4,5)^{T}$. Applying the Frame Generating Algorithm for the multicriteria location problem $(\mathrm{P})$, we have the frame of $Q E(D)$ illustrated in Figure 4. 


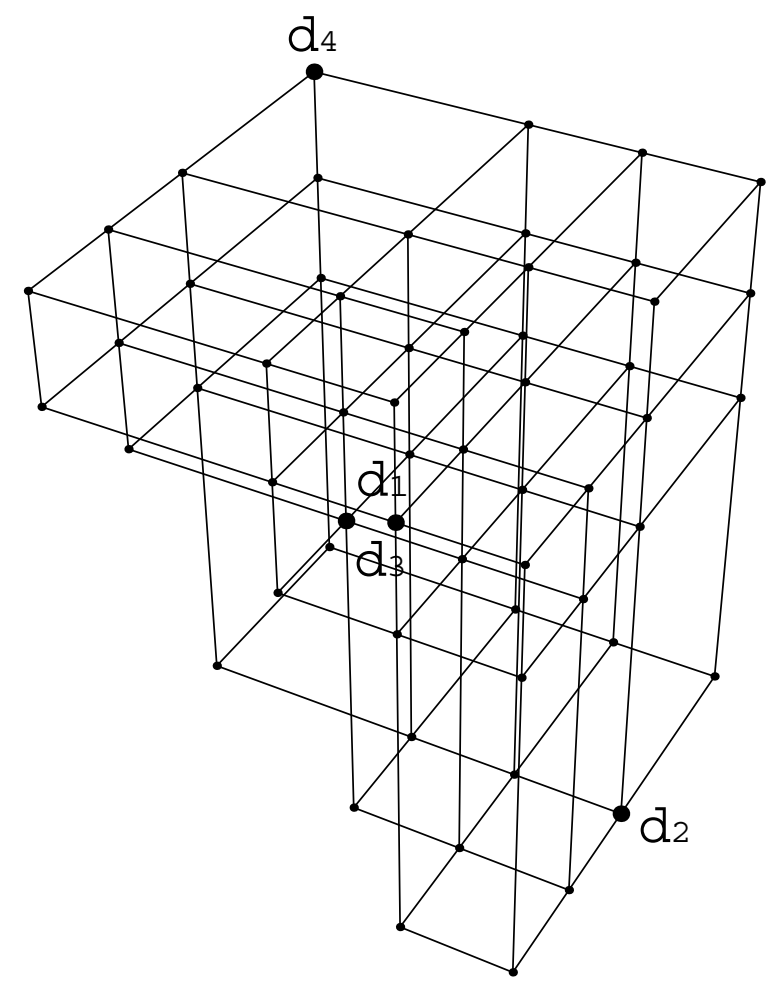

Figure 4: The frame of $Q E(D)(\bullet$ : intersection points in $Q E(D))$

\section{Conclusions}

We dealt with multicriteria and minisum location problems with rectilinear norm in $\mathbb{R}^{3}$. Our main interest was to find $Q E(D)$. First, as corollaries of Theorem 3.1 and 3.2, we obtained characterizations of quasiefficient solutions of $(\mathrm{P})$ by using optimal solutions of $\left(\mathrm{P}_{\boldsymbol{\lambda}}\right)$. They guarantee that $Q E(D)$ can be determined by the frame of $Q E(D)$ and that the frame of $Q E(D)$ is connected. Next, we introduced the concept of the summary diagram to check that an intersection point is quasiefficient in $(\mathrm{P})$ or not. By Theorem 4.1 or Corollary 4.1, we can check that an intersection point is quasiefficient in $(\mathrm{P})$ or not according to the pattern of its summary diagram. Finally, based on these results, we proposed the Frame Generating Algorithm to find the frame of $Q E(D)$. The Frame Generating Algorithm generates the frame of $Q E(D)$ by tracing one-dimensional boxes in $Q E(D)$.

In $\mathbb{R}^{n}(n>3)$, if it can be checked that a given (intersection) point is quasiefficient or not, then our framework is available. However, it is impossible to apply our summary diagrams to check it. Thus, future research could be conducted to check it.

\section{References}

[1] A.V. Aho, J.E. Hopcroft and J.D. Ullman: The Design and Analysis of Computer Algorithms (Addison-Wesley, Reading MA, 1974).

[2] L.G. Chalmet, R.L. Francis and A. Kolen: Finding efficient solutions for rectilinear distance location problems efficiently. European Journal of Operational Research, 6 (1981), 117-124.

[3] Z. Drezner and G.O. Wesolowsky: The asymmetric distance location problem. Transportation Science, 23 (1989), 201-207. 
[4] J.-B. Hiriart-Urruty and C. Lemaréchal: Convex Analysis and Minimization Algorithms I: Fundamentals (Springer-Verlag, Berlin, 1993).

[5] M. Kon: Efficient solutions for multicriteria location problems under the block norm. Mathematica Japonica, 47 (1998), 295-303.

[6] M. Kon: Efficient solutions of multicriteria location problems with rectilinear norm in $\boldsymbol{R}^{3}$. Scientiae Mathematicae Japonicae, 54 (2001), 289-299.

[7] M. Kon: Public opinion survey on home education: application of location problems with rectilinear norm. Scientiae Mathematicae Japonicae, 58 (2003), 99-111.

[8] M. Kon: Efficient solutions of multicriteria location problems with rectilinear norm in $\mathbb{R}^{n}$. Bulletin of the Faculty of Science and Technology, Hirosaki University, 7 (2004), 21-30.

[9] M. Kon and S. Kushimoto: Efficient solutions for multicriteria location problems under the block norm II: application to the development of new products. Scientiae Mathematicae, 1 (1998), 133-140.

[10] T.J. Lowe, J.-F. Thisse, J.E. Ward and R.E. Wendell: On efficient solutions to multiple objective mathematical programs. Management Science, 30 (1984), 1346-1349.

[11] S. Nickel and J. Puerto: Location Theory (Springer-Verlag, Berlin, 2005).

[12] R.T. Rockafellar: Convex Analysis (Princeton University Press, Princeton, N. J., 1970).

[13] A.M. Rodríguez-Chía and J. Puerto: Geometrical description of the weakly efficient solution set for multicriteria location problems. Annals of Operations Research, 111 (2002), 181-196.

[14] J.E. Ward and R.E. Wendell: Characterizing efficient points in location problems under one-infinity norm. In J.-F. Thisse and H.G. Zoller (eds.): Locational Analysis of Public Facilities (North Holland, Amsterdam, 1983), 413-429.

[15] R.E. Wendell, A.P. Hurter, Jr. and T.J. Lowe: Efficient points in location problems. AIIE Transactions, 9 (1977), 238-246.

Masamichi Kon

Graduate School of Science and Technology

Hirosaki University

3 Bunkyo, Hirosaki 036-8561, Japan

E-mail: masakon@cc.hirosaki-u.ac.jp 